\title{
High-density lipoprotein cholesterol to low- density lipoprotein cholesterol ratio in early assessment of disease severity and outcome in patients with acute pancreatitis admitted to the ICU
}

Qin Wu, Xi Zhong, Min Fu, Hao Yang, Hong Bo, Xuelian Liao, Zhi Hu, Bo Wang, Zhongwei Zhang, Xiaodong Jin and Yan Kang ${ }^{*}$ (D)

\begin{abstract}
Background: Patients with acute pancreatitis usually exhibit dyslipidemia and oxidative stress. However, the significance of high-density lipoprotein cholesterol (HDL-C) level, low-density lipoprotein cholesterol (LDL-C) level and the $\mathrm{HDL}-\mathrm{C} / \mathrm{LDL}-\mathrm{C}$ ratio $(\mathrm{H} / \mathrm{L}$ ratio) as markers for disease progression remain unknown.

Aim: The aim of this study was to evaluate the role of HDL-C levels, LDL-C levels and the $H / L$ ratio as markers of disease progression in patients admitted to the intensive cate unit with acute pancreatitis.

Methods: This retrospective study was conducted at a tertiary critical care center in China. Plasma HDL-C and LDL$C$ levels were measured in 166 patients with acute pancreatitis. The associations between HDL-C, LDL-C, H/L ratio, as well as other inflammatory index and mortality, were analyzed. Multivariate cox analysis based on two models was used to determine the independent prognostic factor. Predictive ability of in-hospital mortality for variables was determined using the receiver operating characteristics curves.

Results: Significantly higher $\mathrm{H} / \mathrm{L}$ ratios at admission were observed in patients with acute pancreatitis who died compared with survivors ( 0.93 vs. $0.64, p<0.001)$. The area under the ROC curve for $\mathrm{H} / \mathrm{L}$ ratio-based prediction of mortality was 0.658 . When clinical confounders were included in multivariable cox regression analysis, the association was preserved (Model A HR $=1.587, p=0.011$; Model $\mathrm{BHR}=1.332, p=0.032$ ). The mortality risk in different groups defined by an $\mathrm{H} / \mathrm{L}$ ratio cutoff value was significantly different, based on survival curve analysis.

Conclusion: The H/L ratio at the time of admission to the ICU appears to be a biomarker of disease progression in patients with acute pancreatitis.
\end{abstract}

Keywords: Acute pancreatitis, High-density lipoprotein cholesterol, low-density lipoprotein cholesterol, Mortality

* Correspondence: kangyan@scu.edu.cn

Department of Critical Care Medicine, West China Hospital, Sichuan

University, Chengdu, China

C C The Author(s). 2020 Open Access This article is licensed under a Creative Commons Attribution 4.0 International License, which permits use, sharing, adaptation, distribution and reproduction in any medium or format, as long as you give appropriate credit to the original author(s) and the source, provide a link to the Creative Commons licence, and indicate if changes were made. The images or other third party material in this article are included in the article's Creative Commons licence, unless indicated otherwise in a credit line to the material. If material is not included in the article's Creative Commons licence and your intended use is not permitted by statutory regulation or exceeds the permitted use, you will need to obtain permission directly from the copyright holder. To view a copy of this licence, visit http://creativecommons.org/licenses/by/4.0/. The Creative Commons Public Domain Dedication waiver (http://creativecommons.org/publicdomain/zero/1.0/) applies to the data made available in this article, unless otherwise stated in a credit line to the data. 


\section{Background}

Acute pancreatitis (AP), an inflammatory disease of the pancreas, is the leading cause of admission to hospital for gastrointestinal disorders in China and many other countries $[1,2]$. Characterized by local and systemic inflammation, the severity of AP can range from mild to severe [3]. Although most patients with AP have the mild form of the disease, patients with moderate to severe AP, especially those who are admitted to the intensive care unit (ICU) due to organ failure, constitute a substantial burden to the Chinese medical system [4, 5]. Early assessment of disease severity and prognosis is crucial in defining treatment strategies, because effective treatment can significantly reduce mortality in patients with AP [6].

Systemic inflammation in patients with AP induces the release of free oxygen radicals through an immunemediated mechanism that has been suggested to contribute to the pathogenesis of inflammatory diseases, and is believed to be associated with lipid profile changes due to impaired pancreatic exocrine secretion and endocrine function [7]. Recently, the relationship between lowdensity lipoprotein cholesterol (LDL-C), high-density lipoprotein cholesterol (HDL-C), cholesterol levels and pancreatic function has garnered much attention [8]. The HDL-C/LDL-C ratio (H/L ratio) is a powerful risk predictor and has been used as a biomarker not only for cardiovascular disease but also for ankylosing spondylitis and other diseases $[9,10]$. However, the relationship between HDL-C and LDL-C levels, H/L ratio and outcome in patients with AP has not been confirmed. In this study, we aimed to investigate the association of LDL-C levels, HDL-C levels and H/L ratio with severity and outcome in patients with AP, comparing their predictive value with the traditional predictive values.

\section{Methods}

\section{Study population}

This was a retrospective database cohort study that was conducted in Department of Critical Care Medicine at West China Hospital in China. The study was approved by the institutional review board of West China Hospital. Informed consent from individuals was waived due to the retrospective, observational and anonymous nature of the study. The study included consecutive patients admitted to the Department of Critical Care Medicine at West China Hospital between January 2016 and December 2017. We prospectively established a patient database for our study, and information was collected automatically by computers or manually by attending physicians. The integrity of the data was monitored using a specialized system (Clinical Information System for Intensive Care Practitioners, Sichuan Smart Medical Information Systems, Chengdu, China).

\section{Study protocol}

First, we scanned medical records from all patients discharged from ICU during the study period for a diagnosis of AP. The AP was diagnosed based on a combination of symptoms, physical examination and key laboratory values. Two of the following three characteristics were required: 1) upper abdominal pain during acute episodes, 2) serum amylase or lipase levels three times above normal levels, and 3) abdominal imaging findings consistent with AP. The exclusion criteria were as follows: a recurrence of chronic pancreatitis, the inability to obtain laboratory measurements or medical records, hospitalization more than $48 \mathrm{~h}$ after onset of abdominal pain, age under 18, and stay in the ICU of less than $24 \mathrm{~h}$. For patients with more than one stay in the ICU during the study period, the first episode was retained for further analysis. Three levels of severity were defined according to the revised Atlanta classification of AP. For the etiological diagnosis, gallstonerelated AP can be diagnosed if the images (computed tomography, magnetic resonance imaging or ultrasonography) show the definitive presence of gallstones. Hyperlipidemic pancreatitis is defined as severe serum hyperlipidemia when triglyceride levels are greater than $11.3 \mathrm{mmol} / \mathrm{L}$, or in excess of $5.65 \mathrm{mmol} / \mathrm{L}$ in patients with grossly lipemic serum. A patient would be diagnosed as having alcoholic pancreatitis if the patient had a history of alcohol use before the AP episode and consumed more than $50 \mathrm{~g} /$ day for more than 5 years [11].

\section{Data collection}

The data that are recorded routinely in our hospital at admission include the severity of the disease. The Acute Physiology and Chronic Health Evaluation II (APACHE II) score and Ranson's score were calculated by the attending physician who saw the patient at admission. Cholesterol, HDL-C and LDL-C levels were evaluated by laboratory tests after admission and whenever required according to the judgment of the attending physician (available $24 \mathrm{~h} /$ day). Arterial was collected for air blood gas analysis whenever necessary. Usually, laboratory tests are performed within $4 \mathrm{~h}$ of admission and evaluated at least once per day during an ICU stay. Laboratory values were measured within $2 \mathrm{~h}$ after blood collection in the Department of Laboratory Medicine at West China Hospital. Cholesterol, HDL-C and LDL-C levels were measured directly using a Biochemical Analyzer (Dimension AR/AVL Clinical Chemistry System, Newark, NJ, USA). Other laboratory parameters obtained as part of our study included red blood cell count (RBC), hemoglobin (HGB), hematocrit (HCT), platelet count (PLT), white blood cell count (WBC); activated partial thromboplastin time (APTT), fibrinogen (Fib), thrombin time (TT), arterial oxygen partial pressure $(\mathrm{PaO} 2)$, arterial carbon 
dioxide partial pressure $(\mathrm{PaCO} 2)$, lactate $(\mathrm{Lac})$, procalcitonin (PCT),c-reactive protein (CRP), interleukin 6 (IL6), total bilirubin (TB), direct bilirubin (DB), alanine aminotransferase (ALT), aspartate aminotransferase (AST), creatinine (Crea), albumin (ALB), glucose (Glu), cystatin $\mathrm{C}$ (Cys-c), gamma-glutamyl transferase (GGT), LDL-C, HDL-C and TG.

\section{Statistical analyses}

We analyzed our data using SPSS Statistics 25 (IBM Corp., Armonk, NY, USA) and GraphPad Prism (GraphPad Software, San Diego, CA, USA), with significance defined as $P<0.05$. Continuous variables were presented as means \pm standard deviation (SD) or median and interquartile range (IQR) and tested for distribution normality using the Kolmogorov-Smirnov test. Categorical variables are expressed as percentages. The Student's ttest or Mann-Whitney $U$ was used for quantitative data based on normality assumption. Categorical variables were analyzed in using the Fisher exact test. Pearson's correlation analysis was used to assess the correlation between variables. A multivariable cox regression analysis was performed to examine the association of variables with the risk of death after adjustment for potential confounders (Model A for age, gender and etiology, underlying medical conditions and severity; Model $\mathrm{B}$ for age, gender and etiology, underlying medical conditions, severity, APACHE II score and Ranson's score). Results of regression analysis were demonstrated as hazards ratio with corresponding 95\% confidence interval (CI). Receiver operating characteristics (ROC) curves were constructed and the area under the curve (AUC) as well as the corresponding 95\% CI was assessed as a summary measure of predictive accuracy of variables for mortality. Log-rank tests were used to assess the statistical difference between the survival curves constructed using the Kaplan-Meier method.

\section{Results}

A total of 591 records from patients diagnosed with pancreatitis and discharged from the ICU during our study period were initially included. For patients who had more than one ICU stay during the study period, the medical records from the first stay only were retained, resulting in the elimination of 91 records from readmitted patients. Of the 500 unique patients, 22 were hospitalized for recurrence of chronic pancreatitis and 283 were admitted to hospital for postoperative necrosis or pseudocysts caused by previous episodes of AP. In addition, 10 patients were younger than 18 years of age at admission. Fourteen patients were in the ICU for less than $24 \mathrm{~h}$, and five were excluded due to insufficient data. Ultimately, the records for 166 patients with AP were retained for further analysis (Fig. 1). The baseline

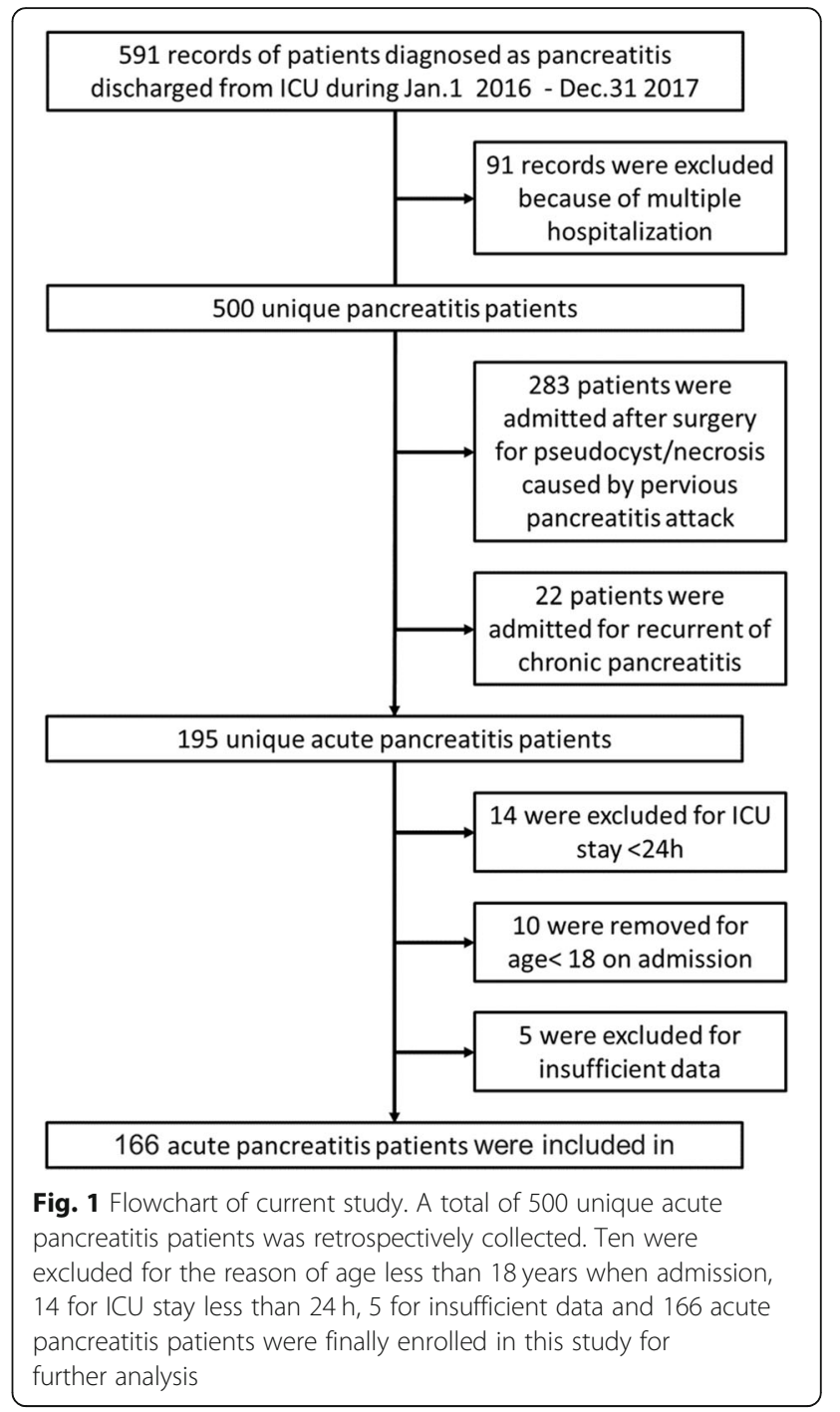

characteristics of the study population are summarized in Table 1 and Supplementary Table 1 . The average age of the entire cohort was $47.49 \pm 15.06$ years, and there were more men than women. Gallstones were the most common cause of AP in these patients. The average APACHE II score and Ranson's score were 19.27 \pm 7.32 and $4.24 \pm 1.55$, respectively. Severe AP was the most common type of AP observed in this cohort. The total in-hospital mortality rate was $27.11 \%$, and the mean ICU stay was $23.37 \pm 22.40$ days.

We divided our AP patients into two groups based on whether or not they survived the hospital stay. There was no statistical difference in the age or gender of survivors and non-survivors (Table 1). However, a significant difference was observed between these patients in terms of etiology $(p=0.007)$, APACHE II score $(p<$ $0.001)$, HDL-C level $(p<0.001)$, LDL-C level $(p<0.001)$, $\mathrm{H} / \mathrm{L}$ ratio $(p<0.001), \operatorname{RBC}(p=0.001)$, HGB $(p=0.001)$, HCT $(p=0.001)$, TB $(p=0.003)$, DB $(p=0.002)$ and Cys- 
Table 1 Demographics, Clinical and Outcome data of acute pancreatitis patient cohort

\begin{tabular}{|c|c|c|c|c|}
\hline Parameters & All $(\boldsymbol{n}=166)$ & Non-Survivor $(\boldsymbol{n}=45)$ & Survivor $(\boldsymbol{n}=121)$ & $P$ \\
\hline \multicolumn{5}{|l|}{ Demographic Data } \\
\hline Age, mean (SD),y & $47.49(15.06)$ & $49.67(13.73)$ & $46.68(15.53)$ & 0.310 \\
\hline Male, n (\%) & $97(58.43)$ & $25(55.60)$ & $72(59.50)$ & 0.646 \\
\hline Etiology, n (\%) & & & & 0.007 \\
\hline Biliary & $71(42.80)$ & $15(33.30)$ & $56(46.30)$ & \\
\hline Hyperlipidemia & $34(20.50)$ & $5(11.10)$ & $29(24.00)$ & \\
\hline Others & $61(36.70)$ & $25(55.60)$ & $36(29.80)$ & \\
\hline \multicolumn{5}{|l|}{ Underlying medical conditions } \\
\hline Hypertension, n (\%) & $24(14.50)$ & $5(11.10)$ & $19(15.70)$ & 0.455 \\
\hline Diabetes Mellitus, n (\%) & $28(16.90)$ & $5(11.10)$ & $23(19.00)$ & 0.227 \\
\hline Severity, n (\%) & & & & 0.533 \\
\hline Moderately severe & $27(16.30)$ & $6(13.30)$ & $21(17.40)$ & \\
\hline Severe & $139(83.70)$ & $39(86.70)$ & $100(82.60)$ & \\
\hline APACHEIIScore ${ }^{a}$, mean (SD) & $19.27(7.32)$ & $22.53(5.69)$ & $18.03(7.50)$ & $<0.001$ \\
\hline Ranson's Score ${ }^{a}$, mean (SD) & $4.24(1.55)$ & $4.29(1.61)$ & $4.11(1.36)$ & 0.493 \\
\hline $\mathrm{CHOL}^{\mathrm{a}}$, mean $(\mathrm{SD}), \mathrm{mmol} / \mathrm{L}$ & $3.41(2.79)$ & $3.00(3.42)$ & $3.00(3.42)$ & 0.257 \\
\hline $\mathrm{HDL}-\mathrm{C}^{\mathrm{a}}$, mean $(\mathrm{SD}), \mathrm{mmol} / \mathrm{L}$ & $0.39(0.28)$ & $0.26(0.13)$ & $0.44(0.31)$ & $<0.001$ \\
\hline LDL-Ca, mean (SD), mmol/L & $0.91(0.70)$ & $0.54(0.53)$ & $1.05(0.71)$ & $<0.001$ \\
\hline $\mathrm{H} / \mathrm{L}$ ratio ${ }^{a}$, mean (SD) & $0.72(0.92)$ & $0.93(1.29)$ & $0.64(0.72)$ & $<0.001$ \\
\hline Statins usage ${ }^{* *}, \mathrm{n}(\%)$ & $2(1.20)$ & $1(2.22)$ & $1(0.82)$ & 0.464 \\
\hline Ventilation free days ${ }^{b}$, mean (SD), d & $16.87(8.93)$ & $13.64(9.16)$ & $18.07(8.56)$ & 0.004 \\
\hline Renal replacement therapy, n (\%) & $27(16.30)$ & $12(26.70)$ & $15(12.40)$ & 0.149 \\
\hline Hospital cost, mean (SD), CHY & $182,392.76(141,028)$ & $229,767(163.568)$ & $164,627(127,869)$ & 0.008 \\
\hline ICU LOS, mean (SD), d & $23.37(22.40)$ & $20.91(16.56)$ & $24.28(24.22)$ & 0.391 \\
\hline Hospital LOS, mean (SD), d & $30.54(23.30)$ & $23.27(17.18)$ & $33.27(24.73)$ & 0.014 \\
\hline
\end{tabular}

APACHE Acute Physiology and Chronic Health Evaluation, CHOL cholesterol, HDL-C High-density lipoprotein cholesterol, LDL-C Low-density lipoprotein cholesterol, $H / L$ HDL-C/ LDL-C, LOS length of stay

a on admission

${ }^{b}$ within 28 days

C $(p<0.001)$. Multivariate Cox analysis based on two models showed that the $\mathrm{H} / \mathrm{L}$ ratio was an independent prognostic factor for patients with AP (Table 2, Fig. 2). Moreover, in patients with gallstone AP, the $\mathrm{H} / \mathrm{L}$ ratio was significantly different between groups (Supplementary Material 2). In addition, there was a significant correlation between the $\mathrm{H} / \mathrm{L}$ ratio and the APACHE II score $(p<0.001, \mathrm{r}=0.445)$ and Ranson's score $(p=0.018$, $\mathrm{r}=0.229$ ). In addition, the trend analysis shown in Table 3 indicated that an increased $\mathrm{H} / \mathrm{L}$ ratio was associated with increased in-hospital mortality.

We then compared the diagnostic performances of APACHE II score, Ranson's score, Lac, PCT, CRP, IL-6 and $\mathrm{H} / \mathrm{L}$ ratio using the AUC for discrimination mortality. The AUCs for APACHE II score, Ranson's score, Lac, PCT, CRP, IL-6 and H/L ratio were 0.694 ([95\% CI] 0.604-0.781), 0.533 ([95\% CI] 0.458-0.619), 0.546 ([95\% CI] 0.469-0.659), 0.603 ([95\% CI] 0.529-0.715), 0.572 ([95\% CI $]$ 0.484-0.673), $0.646([95 \% \mathrm{CI}] \quad 0.563-0.745)$
Table 2 Multivariate Cox analysis for HDL-C, LDL-C and H/L ratio associated with in-hospital mortality

\begin{tabular}{|c|c|c|c|c|}
\hline \multirow[t]{2}{*}{ variables } & \multirow{2}{*}{$\begin{array}{l}\text { Hazard } \\
\text { Ratio }\end{array}$} & \multicolumn{2}{|c|}{$95 \% \mathrm{Cl}$} & \multirow{2}{*}{$\begin{array}{l}P \\
\text { value }\end{array}$} \\
\hline & & lower & $\overline{\text { upper }}$ & \\
\hline \multicolumn{5}{|l|}{ Model A } \\
\hline $\mathrm{HDL}-\mathrm{C}$ & 0.322 & 0.218 & 0.815 & 0.010 \\
\hline LDL-C & 0.421 & 0.119 & 0.754 & 0.024 \\
\hline $\mathrm{H} / \mathrm{L}$ ratio & 1.587 & 1.169 & 2.146 & 0.011 \\
\hline \multicolumn{5}{|l|}{ Model B } \\
\hline HDL-C & 0.553 & 0.279 & 1.099 & 0.091 \\
\hline LDL-C & 0.628 & 0.262 & 1.168 & 0.132 \\
\hline $\mathrm{H} / \mathrm{L}$ ratio & 1.332 & 1.133 & 1.985 & 0.032 \\
\hline
\end{tabular}

Model $\mathrm{A}$ adjust for age, gender and etiology, underlying medical conditions and severity;

Model $B$ adjust for age, gender and etiology, underlying medical conditions, severity, APACHE II score and Ranson's score

HDL-C High-density lipoprotein cholesterol, LDL-C Low-density lipoprotein cholesterol, H/L HDL-C/ LDL-C

95\% Cl: $95 \%$ confidence interval 


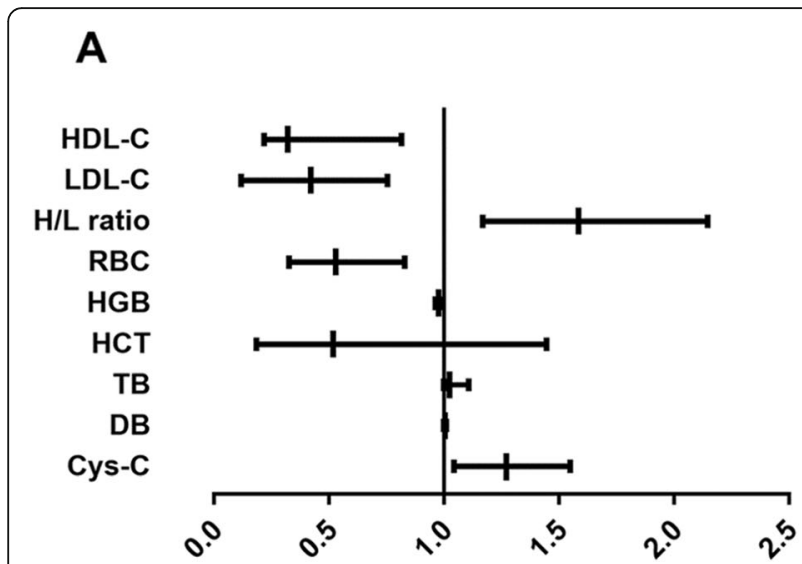

\section{B}

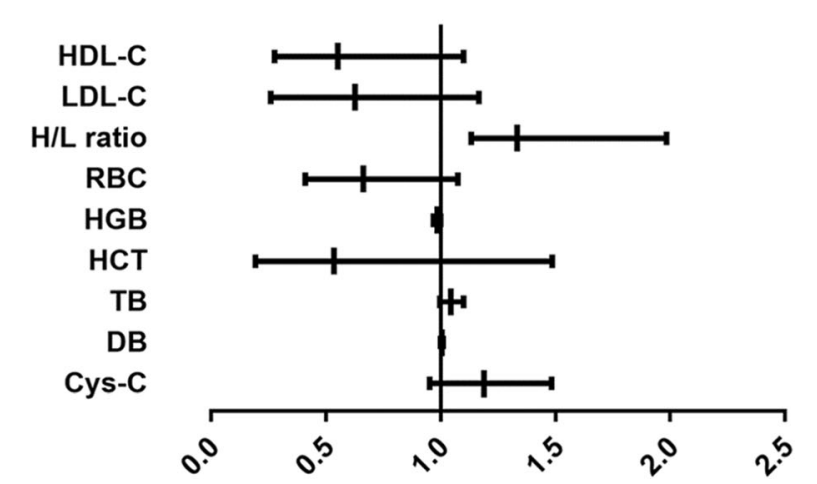

Fig. 2 Forest plots from multivariate Cox regression models: a adjust for age, gender and etiology, underlying medical conditions and severity and (b) adjust for age, gender and etiology, underlying medical conditions, severity, APACHE II score and Ranson's score. RBC: Red blood cell, HGB: Hemoglobin, HCT: Hematocrit, TB: Total bilirubin, DB: Direct bilirubin, Cys-c: Cystatin C, HDL-C: High-density lipoprotein cholesterol, LDL-C: Low-density lipoprotein cholesterol, H/L: HDL-C/ LDL-C

and 0.658 ([95\% CI] 0.564-0.747), respectively (Fig. 3). Kaplan-Meier survival curves were established based on calculating the minimum $\mathrm{d}(\mathrm{H} / \mathrm{L}$ ratio, 0.465$)$ using cutoff values determined from the receiver operating characteristic curves. After stratification according to $\mathrm{H} / \mathrm{L}$ ratio, the 28-day survival rates for patients with AP were significantly different (Fig. 4). Patients with a lower H/L ratio at admission had a higher survival rate than patients with a higher $\mathrm{H} / \mathrm{L}$ ratio (log-rank test, $p<0.001$, Table 4). In addition, using this threshold (H/L ratio, $0.465)$, the sensitivity of the $\mathrm{H} / \mathrm{L}$ ratio in discriminating between non-survivors and survivors was $71.10 \%$, with a specificity of $57.0 \%$.

\section{Discussion}

In this study, we evaluated the relationship between lipid levels at the time of admission and hospital mortality in patients with AP. We found that higher $\mathrm{H} / \mathrm{L}$ ratio levels were positively correlated with increased mortality in patients with AP, and determined the optimal cutoff for using the $\mathrm{H} / \mathrm{L}$ ratio to assess AP-related mortality. The main results from this study indicate that the $\mathrm{H} / \mathrm{L}$ ratio can be used to predict outcomes in patients with AP. To the best of our knowledge, this is the first study to confirm the predictive value of the $\mathrm{H} / \mathrm{L}$ ratio and analyze the accuracy of this indicator in patients with AP.

Cholesterol is a structural component of cell membranes and is localized in membrane micro-domains that assemble signal transduction machinery. It is associated with proteins involved in key cell signaling pathways that are closely related to inflammation $[8,12]$. There are five major lipoproteins: chylomicrons and very low-density lipoprotein, medium-density lipoprotein, low-density lipoprotein and high-density lipoprotein, which are classified compared with the density of the surrounding water $[13,14]$. To facilitate cell absorption and utilization of fat through receptor-mediated endocytosis, LDL carries all of the fatty molecules, cholesterol, phospholipids and triglycerides, while HDL transports cholesterol from the cells and tissues back to the liver [15]. Rather than measuring actual HDL and LDL particles, assessing HDL-C and LDL-C levels provides information about how much cholesterol is delivered by all HDL and LDL particles. HDL and LDL oxidation is associated

Table 3 Outcomes of patients stratifying based on $\mathrm{H} / \mathrm{L}$ ratio quartile

\begin{tabular}{llllll}
\hline Parameters & $\begin{array}{l}\text { Patient } \\
\text { number }\end{array}$ & $\begin{array}{l}\text { In } \\
\text { hospital } \\
\text { death }\end{array}$ & Adjusted Hazard Ratio (95\% Cl) & Model 2 & ${\text { Model } 3^{\mathbf{c}}}^{\mathbf{b}}$ \\
\hline Quartile 1 & 41 & 6 & 1.00 (reference) & 1.00 (reference) & 1.00 (reference) \\
Quartile 2 & 42 & 9 & $1.573(1.190-1.848)$ & $1.539(0.985-1.815)$ & $0.784(0.245-2.559)$ \\
Quartile 3 & 41 & 14 & $2.844(1.691-4.920)$ & $1.777(1.045-4.774)$ & $1.796(0.681-5.731)$ \\
Quartile 4 & 42 & 16 & $4.188(1.832-5.754)$ & $2.144(1.804-5.720)$ & $2.077(0.753-5.733)$ \\
P for trend & & & 0.006 & 0.022 & 0.039 \\
\hline
\end{tabular}

\footnotetext{
${ }^{a}$ Crude, no adjustment
}

${ }^{\mathrm{b}}$ Adjusted for age, gender, etiology, underlying medical conditions, and severity

c Adjusted for age, gender, etiology, underlying medical conditions, severity, APACHE II score and Ranson's score 

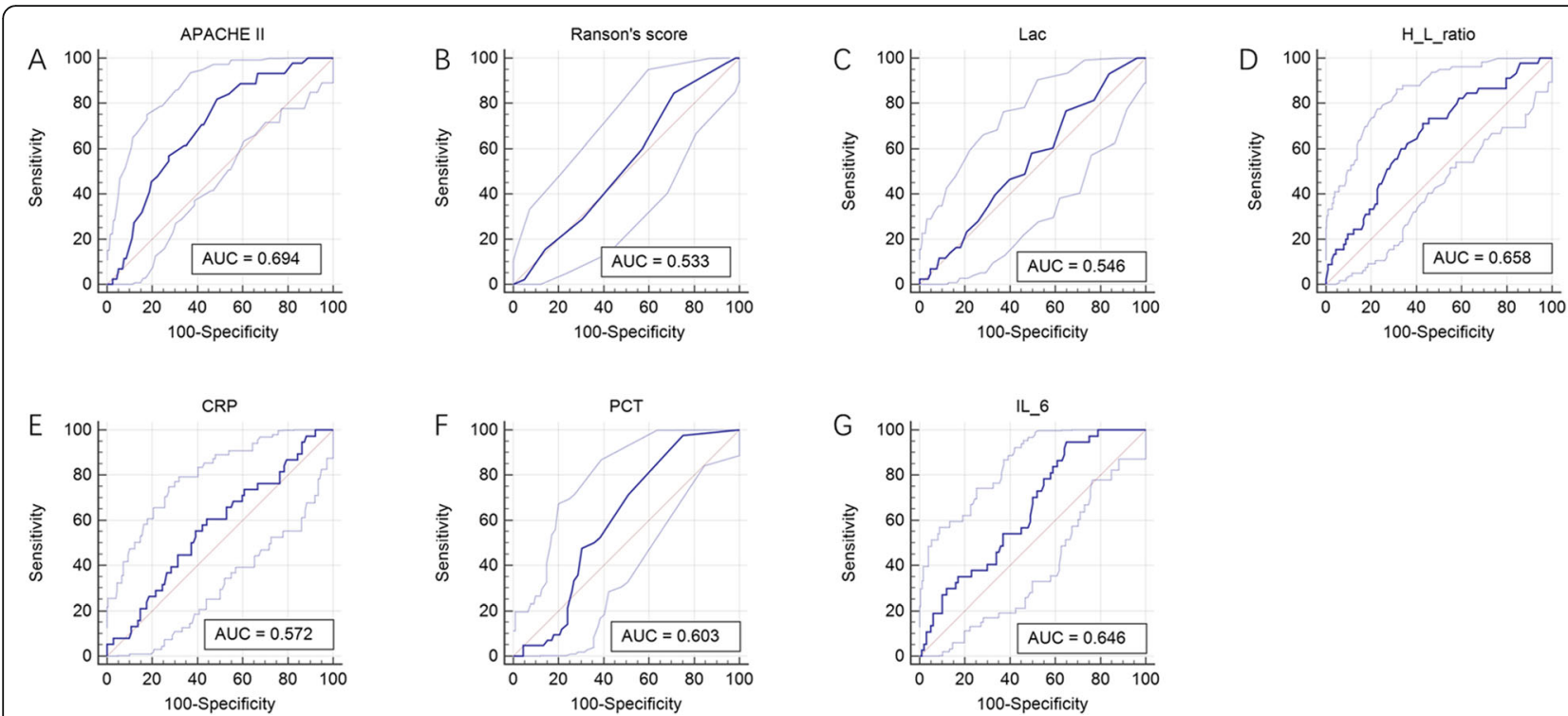

Fig. 3 Receiver operating characteristic (ROC) curves of the APACHE II score, Ranson's score, lactate, H/L ratio, C-reactive protein, procalcitonin, and interleukin 6 level in predicting mortality after ICU admission in acute pancreatitis patients

with an increased risk of developing cardiovascular disease because the oxidized forms are more susceptible to proteoglycan [16].

The relationship between HDL-C, LDL-C and cardiovascular disease has been well established over the years. Many guidelines recommend lowering LDL-C to reduce the risk of coronary heart disease, and highlight the protective role of HDL-C [17]. Given the essential function of blood lipids, LDL-C and HDL-C levels can be used to predict mortality not only in patients with cardiovascular disease but also in patients with colorectal cancer [18]. Although pancreatic exocrine function is directly linked to lipid metabolism, little is known about the predictive value of HDL-C and LDL-C in patients with AP.

In this retrospective study, we analyzed medical records from 166 patients with AP, who were admitted to the ICU for organ support, and evaluated the role of LDL-C and HDL-C levels and H/L ratios in predicting mortality in these patients. Interestingly, we found that

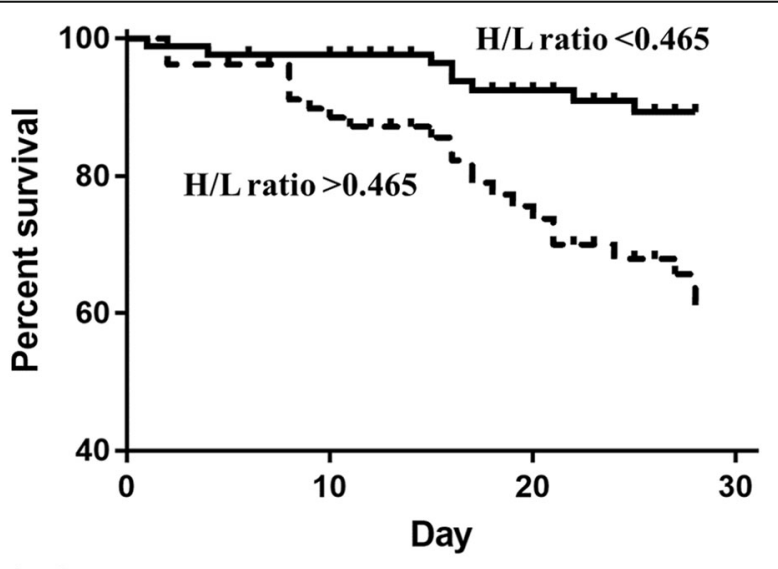

Numbers of survival

$\begin{array}{lllll}\text { H/L ratio }<0.465 & 86 & 84 & 80 & 78 \\ \text { H/L ratio }>0.465 & 80 & 72 & 63 & 56\end{array}$

Fig. 4 Survival analysis between groups based on an $\mathrm{H} / \mathrm{L}$ ratio level cutoff. Kaplan-Meier survival curves were created based on $\mathrm{H} / \mathrm{L}$ ratio cutoff values of 0.465 . A significant difference was observed between the two curves 
Table 4 Clinical data of the acute pancreatitis patient cohort based on $\mathrm{H} / \mathrm{L}$ ratio

\begin{tabular}{|c|c|c|c|}
\hline Parameters & $\mathrm{H} / \mathrm{L}$ ratio $<0.465(\boldsymbol{n}=86)$ & $\mathrm{H} / \mathrm{L}$ ratio $>0.465(\boldsymbol{n}=80)$ & $P$ \\
\hline \multicolumn{4}{|l|}{ Demographic Data } \\
\hline Age, mean (SD),y & $45.71(13.67)$ & $49.18(16.20)$ & 0.141 \\
\hline Male, n (\%) & $45(52.33)$ & $52(65.00)$ & 0.098 \\
\hline Etiology, n (\%) & & & 0.031 \\
\hline Biliary & $36(41.86)$ & $35(43.80)$ & \\
\hline Hyperlipidemia & $24(27.91)$ & $10(12.50)$ & \\
\hline Others & $26(30.23)$ & $35(43.80)$ & \\
\hline \multicolumn{4}{|l|}{ Underlying medical conditions } \\
\hline Hypertension, n (\%) & $6(6.97)$ & $18(22.50)$ & 0.004 \\
\hline Diabetes Mellitus, n (\%) & $14(16.28)$ & $14(17.50)$ & 0.834 \\
\hline Severity, n (\%) & & & 0.034 \\
\hline Moderately severe & $19(22.09)$ & $8(10.00)$ & \\
\hline Severe & $67(77.91)$ & $72(90.00)$ & \\
\hline APACHEIIScore*, mean (SD) & $17.99(6.50)$ & $20.50(7.85)$ & 0.027 \\
\hline Ranson's Score*, mean (SD) & $4.18(1.46)$ & $4.30(1.64)$ & 0.635 \\
\hline $\mathrm{CHOL}$, mean (SD), mmol/L & $3.63(2.61)$ & $3.20(2.96)$ & 0.323 \\
\hline $\mathrm{HDL}-\mathrm{C}$, mean (SD), mmol/L & $0.33(0.17)$ & $0.44(0.36)$ & 0.015 \\
\hline LDL-C, mean (SD), mmol/L & $1.25(0.72)$ & $0.59(0.51)$ & $<0.001$ \\
\hline $\mathrm{H} / \mathrm{L}$ ratio, mean (SD) & $0.28(0.09)$ & $1.14(1.15)$ & $<0.001$ \\
\hline Ventilation free days\#, mean (SD), d & $16.13(9.16)$ & $17.57(8.71)$ & 0.045 \\
\hline Renal replacement therapy, n (\%) & $6(6.97)$ & $21(26.25)$ & 0.001 \\
\hline ICU LOS, mean (SD), d & $25.86(26.62)$ & $21.02(17.43)$ & 0.163 \\
\hline Hospital LOS, mean (SD), d & $33.40(22.88)$ & $27.85(23.50)$ & 0.126 \\
\hline \multicolumn{4}{|l|}{ Complete blood count* } \\
\hline $\mathrm{RBC}$, mean $(\mathrm{SD}), \times 10^{9} / \mathrm{L}$ & $3.15(0.69)$ & $3.21(1.00)$ & 0.649 \\
\hline HGB, mean (SD), g/L & $95.34(22.37)$ & $95.12(27.54)$ & 0.955 \\
\hline $\mathrm{HCT}$, mean (SD), L/L & $0.29(0.06)$ & $0.29(0.08)$ & 0.844 \\
\hline PLT, mean (SD), $\times 10^{9} / \mathrm{L}$ & $156.36(104.59)$ & $144.06(111.11)$ & 0.463 \\
\hline WBC, mean $(S D), \times 10^{9} / \mathrm{L}$ & $11.95(5.18)$ & $12.74(6.52)$ & 0.389 \\
\hline \multicolumn{4}{|l|}{ Coagulation test ${ }^{*}$} \\
\hline APTT, mean (SD), s & $36.57(10.40)$ & $44.76(19.51)$ & 0.001 \\
\hline Fib, mean (SD), g/L & $5.07(1.90)$ & $4.13(1.77)$ & 0.001 \\
\hline$\Pi$, mean $(S D), s$ & $19.52(12.51)$ & $18.05(2.65)$ & 0.290 \\
\hline \multicolumn{4}{|l|}{ Arterial Blood Gas Test* } \\
\hline $\mathrm{pH}$, mean (SD) & $7.36(0.27)$ & $7.28(0.46)$ & 0.193 \\
\hline $\mathrm{PaO} 2$, mean (SD), mmHg & $84.18(29.66)$ & $95.73(41.32)$ & 0.045 \\
\hline $\mathrm{PaCO} 2$, mean $(\mathrm{SD}), \mathrm{mmHg}$ & $40.25(6.77)$ & $39.50(9.84)$ & 0.573 \\
\hline $\mathrm{Lac}$, mean (SD), mmol/L & $1.63(0.49)$ & $1.96(1.02)$ & 0.012 \\
\hline \multicolumn{4}{|l|}{ Biochemical analysis* } \\
\hline $\mathrm{PCT}$, mean $(\mathrm{SD}), \mathrm{ng} / \mathrm{mL}$ & $6.82(16.18)$ & $10.44(17.42)$ & 0.184 \\
\hline CRP, mean (SD), mg/L & $189.16(105.01)$ & $183.75(92.43)$ & 0.747 \\
\hline IL-6, mean (SD), ng/mL & $287.52(481.42)$ & $459.90(752.33)$ & 0.114 \\
\hline Serum sodium, mean (SD), mmol/L & $137.66(6.60)$ & $140.05(7.72)$ & 0.035 \\
\hline Serum potassium, mean (SD), mmol/L & $3.75(0.41)$ & $3.81(0.50)$ & 0.368 \\
\hline
\end{tabular}


Table 4 Clinical data of the acute pancreatitis patient cohort based on $\mathrm{H} / \mathrm{L}$ ratio (Continued)

\begin{tabular}{llll}
\hline Parameters & $H / L$ ratio $<0.465(\boldsymbol{n}=86)$ & $H / L$ ratio $>0.465(\boldsymbol{n}=80)$ & $P$ \\
\hline Serum chloride, mean (SD), mmol/L & $108.05(6.33)$ & $109.45(6.95)$ & 0.181 \\
TB, mean (SD), mmol/L & $40.29(57.13)$ & $41.17(53.33)$ & $33.20(46.99)$ \\
DB, mean (SD), mmol/L & $30.97(47.86)$ & $92.73(199.87)$ & 0.918 \\
ALT, mean (SD), mmol/L & $50.06(167.93)$ & $164.60(465.49)$ & 0.763 \\
AST, mean (SD), mmol/L & $78.55(228.91)$ & $29.41(6.21)$ & 0.140 \\
ALB, mean (SD), mmol/L & $30.74(5.11)$ & $10.06(3.72)$ & 0.137 \\
Glu, mean (SD), mmol/L & $10.07(3.11)$ & $212.41(196.63)$ & 0.135 \\
Crea, mean (SD), mmol/L & $141.64(140.04)$ & $1.93(1.35)$ & 0.982 \\
Cys-c, mean (SD), mmol/L & $1.43(0.83)$ & $91.62(108.70)$ & 0.009 \\
GGT, mean (SD), mmol/L & $108.49(123.97)$ & $4.07(5.21)$ & 0.005 \\
TG, mean (SD), mmol/L & $3.56(2.26)$ & 0.352 \\
\hline
\end{tabular}

APACHE Acute Physiology and Chronic Health Evaluation, RBC Red blood cell, HGB Hemoglobin, HCT Hematocrit, MCV Mean corpuscular volume, MCH Mean corpuscular hemoglobin, MCHC Mean corpuscular hemoglobin concentration, RDW Red blood cell distribution width, PLT Platelets, WBC white blood cell, INR International normalized ratio, PT prothrombin time, APTT Activated partial thromboplastin time, Fib fibrinogen, $\Pi$ Thrombin time, PaO2 Arterial oxygen partial pressure, $\mathrm{PaCO} 2$ Arterial carbon dioxide partial pressure, $B E$ Base excess, Lac lactate, $P C T$ Procalcitonin, $T B$ Total bilirubin, $D B$ Direct bilirubin, $A L T$ Alanine Aminotransferase, AST Aspartate Aminotransferase, ALP Alkaline Phosphatase, TP Total protein, ALB Albumin, Glu Glucose, Crea Creatinine, Cys-C Cystatin C, GGT Gamma-Glutamyl Transferase, TG Triglycerides, $C H O L$ cholesterol, HDL-C High-density lipoprotein cholesterol, $L D L-C$ Low-density lipoprotein cholesterol, H/L HDLC/ LDL-C, LOS length of stay, IQR interquartile range

the HDL-C/LDL-C ratio is a potential biomarker, and is more sensitive and specific than lactate, a biomarker that is widely recognized as a marker of mortality in critically ill patients [9].

The mechanism of the relationship between $\mathrm{H} / \mathrm{L}$ ratios and AP prognosis is not clear, but there are several plausible explanations. Some studies have shown that there is some correlation between serum lipid levels and nutritional status, and the predictive value of $\mathrm{H} / \mathrm{L}$ ratios could therefore be attributable to the nutritional status of patients with AP $[9,19]$. However, there was no difference in serum albumin levels between survivors and non-survivors in our study. Therefore, it is unlikely that the different $\mathrm{H} / \mathrm{L}$ ratios observed in these patients were related to nutritional status. Besides, we observe a inconsistent trend of cholesterol levels and triglyceride level in our cohort. Since cholesterol has an impact on maintaining vascular integrity and LDL-C levels can alter platelet acceptability through their effects on platelet activating factor, we hypothesize that the $\mathrm{H} / \mathrm{L}$ ratio has a more complex role in AP progression, and believe that further research regarding this role is urgently needed.

AP may be caused by several factors. Gallstones and alcohol abuse are long-term risk factors, although hypertriglyceridemia is more common in China than alcohol abuse [20]. The method most commonly used to measure LDL-C is the Friedewald equation. Various confounding laboratory abnormalities can be present in patients with AP, especially in those with hypertriglyceridemia pancreatitis, and LDL-C levels are still the main target for treatment in patients with hyperlipidemia and the primary factor on which most of our clinical decisions are based [13]. In addition, in patients with simple gallstone AP, H/L ratios were still significantly different between survivors and non-survivors, suggesting that our conclusion is not related to AP etiology.

We compared the predicted values of common predictive indicators and the $\mathrm{H} / \mathrm{L}$ ratio. In our study, $\mathrm{H} / \mathrm{L}$ ratio had a higher predictive value than PCT, CRP or IL6. Recently, several studies with small sample sizes reported that low levels of HDL-C are associated with high risk of persistent organ failure in AP [21]. Our study confirmed that HDL-C levels could be a predictor for patients with AP, and we found that the $\mathrm{H} / \mathrm{L}$ ratio had a better predictive value than HDL-C levels. However, we found lower HDL-C is beneficial for patients, which is consistent with other studies [22, 23]. One explanation is that due to the retrospective study design, the lipid status parameters in our study are influenced by some patients leading to the distribution of our data is unlikely with others. In the future we still need more welldesigned research to clarify relationships between HDL$\mathrm{C}$ and AP mortality.

Our study had several limitations. First, because of the small sample size and the retrospective evaluation of cases, selection bias may have affected the generalizability of our results. Second, since this was an observational single-center study, the causal role of LDL-C in AP requires further investigation in prospective multicenter validation studies. In addition, we only examined one-time measurements. Therefore, this study did not address the issue of HDL-C and HDL-C variation in individuals. We use morality as our primary outcome instead of organ failure assessment which is not available for all patients because of the study design though it is not an indicator early enough for AP 
patients' stratification in the ICU. There is an urgent need to obtain additional prospective data in consecutive patients to confirm our findings.

\section{Conclusions}

In summary, our results suggest that higher $\mathrm{H} / \mathrm{L}$ ratios are independently associated with poor progression in patients with AP who are admitted to the ICU. Although our study is preliminary, we speculate that serum $\mathrm{H} / \mathrm{L}$ ratios may be a predictive biomarker for mortality in patients with AP.

\section{Supplementary information}

Supplementary information accompanies this paper at https://doi.org/10. 1186/s12876-020-01315-x.

Additional file 1: Supplementary Data 1. Clinical data of acute pancreatitis patient cohort.

Additional file 2: Supplementary Table 2. Demographics, Clinical and Outcome data of gallstone acute pancreatitis patient cohort.

\section{Abbreviations \\ HDL-C: High-density lipoprotein cholesterol; LDL-C: Low-density lipoprotein cholesterol; H/L: HDL-C/LDL-C; AP: Acute pancreatitis; ICU: Intensive care unit; APACHE II: The Acute Physiology and Chronic Health Evaluation II; RBC: Red blood cell count; HGB: Hemoglobin; HCT: Hematocrit; PLT: Platelet count; WBC: White blood cell count; APTT: Activated partial thromboplastin time; Fib: Fibrinogen; TT: Thrombin time; $\mathrm{PaO} 2$ : Arterial oxygen partial pressure; PaCO2: Arterial carbon dioxide partial pressure; Lac: Lactate; PCT: Procalcitonin; CRP: C-reactive protein; IL-6: Interleukin 6; TB: Total bilirubin; DB: Direct bilirubin; ALT: Alanine aminotransferase; AST: Aspartate aminotransferase; Crea: Creatinine; ALB: Albumin; Glu: Glucose; Cys-C: Cystatin C; GGT: Gamma-glutamyl transferase; SD: Standard deviation; \\ IQR: Interquartile range; AUC: Area under the receiver}

\section{Acknowledgements}

Not applicable.

\section{Authors' information (optional)}

Not applicable

\section{Authors' contributions}

QW, XJ and YK designed the whole study. XZ, MF and HY supervised the whole project and performed data analysis. XZ, HB and XL supervised patient diagnosis and recruitment. $\mathrm{OW}$ and $\mathrm{ZH}$ conducted data analyses and drafted the manuscript. BW and ZZ participated in the manuscript writing and contributed to protocol version development, on which this paper is based. All authors critically reviewed the article and approved the final manuscript.

\section{Funding}

The work was supported by grants from the National Natural Science Foundation of China (81801892, 81901998), Wu Jieping Medical Foundation's special funding fund for clinical research (No.320.6750.17570),Fundamental research funds for the Central university (No.2018SCU12036, the postdoctoral foundation of Sichuan University). China Postdoctoral Science Foundation Grant (2018 M643492), and Sichuan Health Commission Research Project (19PJ237). The funders had no role in study design, data collection and analysis, decision to publish, or preparation of the manuscript.

\section{Availability of data and materials}

The datasets generated and analyzed in this article are not publicly available due to health privacy concerns. However, they are available from the corresponding author and will be obtainable by the public when the database construction is complete.

\section{Ethics approval and consent to participate}

The study was approved by the institutional review board of West China Hospital. Informed consent from individuals was waived due to the retrospective, observational and anonymous nature of the study.

\section{Consent for publication}

Not applicable.

\section{Competing interests}

We declare that we do not have any commercial or associative interest that represents a conflict of interest in connection with the work submitted.

Received: 14 February 2019 Accepted: 22 May 2020

Published online: 27 May 2020

\section{References}

1. Lankisch PG, Apte M, Banks PA. Acute pancreatitis. Lancet. 2015;386(9988): 85-96.

2. Italian Association for the Study of the P. Pezzilli R, Zerbi A, Campra D, Capurso G, Golfieri R, Arcidiacono PG, Billi P, Butturini G, Calculli L et al: Consensus guidelines on severe acute pancreatitis. Dig Liver Dis. 2015;47(7): 532-43

3. Banks PA, Bollen TL, Dervenis C, Gooszen HG, Johnson CD, Sarr MG, Tsiotos GG, Vege SS. Acute pancreatitis classification working G: classification of acute pancreatitis--2012: revision of the Atlanta classification and definitions by international consensus. Gut. 2013;62(1):102-11.

4. Machicado JD, Gougol A, Stello K, Tang G, Park Y, Slivka A, Whitcomb DC, Yadav D, Papachristou Gl. Acute Pancreatitis has Long-term Deleterious Effect on Physical Quality of Life. Clin Gastroenterol Hepatol. 2017.

5. Swaroop VS, Chari ST, Clain JE. Severe acute pancreatitis. JAMA. 2004; 291(23):2865-8

6. Ikeura T, Horibe M, Sanui M, Sasaki M, Kuwagata Y, Nishi K, Kariya S, Sawano H, Goto T, Hamada T, et al. Validation of the efficacy of the prognostic factor score in the Japanese severity criteria for severe acute pancreatitis: a large multicenter study. United European Gastroenterol J. 2017;5(3):389-97.

7. Yuan L, Tang M, Huang L, Gao Y, Li X. Risk factors of hyperglycemia in patients after a first episode of acute pancreatitis: a retrospective cohort. Pancreas. 2016.

8. Hirano K, Saito T, Mizuno S, Tada M, Sasahira N, Isayama H, Matsukawa M, Umefune G, Akiyama D, Saito K, et al. Total cholesterol level for assessing pancreatic insufficiency due to chronic pancreatitis. Gut Liver. 2014;8(5):563-8

9. You S, Zhong C, Xu J, Han Q, Zhang X, Liu H, Zhang Y, Shi J, Huang Z, Xiao $\mathrm{G}$, et al. LDL-C/HDL-C ratio and risk of all-cause mortality in patients with intracerebral hemorrhage. Neurol Res. 2016;38(10):903-8.

10. Kucuk A, Uğur Uslu A, Icli A, Cure E, Arslan S, Turkmen K, Toker A, Kayrak M. The LDL/HDL ratio and atherosclerosis in ankylosing spondylitis. Z Rheumatol. 2016;76(1):58-63.

11. Tenner S, Baillie J, DeWitt J, Vege SS. American College of G: American College of Gastroenterology guideline: management of acute pancreatitis. Am J Gastroenterol. 2013;108(9):1400-1415; 1416.

12. Wang SH, Chou YC, Shangkuan WC, Wei KY, Pan YH, Lin HC. Relationship between plasma triglyceride level and severity of Hypertriglyceridemic pancreatitis. PLoS One. 2016:11(10):e0163984.

13. Jialal I, Remaley AT. Measurement of low-density lipoprotein cholesterol in assessment and management of cardiovascular disease risk. Clin Pharmacol Ther. 2014;96(1):20-2.

14. Stein EA, Raal FJ. New therapies for reducing low-density lipoprotein cholesterol. Endocrinol Metab Clin N Am. 2014;43(4):1007-33.

15. Sherbet DP, Garg P, Brilakis ES, Banerjee S. Low-density lipoprotein cholesterol: how low can we go? Am J Cardiovasc Drugs. 2013;13(4):225-32.

16. Leibowitz M, Karpati T, Cohen-Stavi CJ, Feldman BS, Hoshen M, Bitterman H, Suissa S, Balicer RD. Association between achieved lowdensity lipoprotein levels and major adverse cardiac events in patients with stable ischemic heart disease taking statin treatment. JAMA Intern Med. 2016;176(8):1105-13

17. Chen QJ, Lai HM, Chen BD, Li XM, Zhai H, He CH, Pan S, Luo JY, Gao J, Liu F, et al. Appropriate LDL-C-to-HDL-C ratio cutoffs for categorization of cardiovascular disease risk factors among Uygur adults in Xinjiang, China. Int J Environ Res Public Health. 2016;13(2):235. 
18. Liu YL, Qian HX, Qin L, Zhou XJ, Zhang B. Serum LDL-C and LDL-C/HDL-C ratio are positively correlated to lymph node stages in males with colorectal cancer. Hepatogastroenterology. 2011;58(106):383-7.

19. Sharif S, van der Graaf $Y$, Nathoe HM, de Valk HW, Visseren FL, Westerink J, Group SS. HDL cholesterol as a residual risk factor for vascular events and all-cause mortality in patients with type 2 diabetes. Diabetes Care. 2016; 39(8):1424-30

20. Shen X, Ke L, Yang D, Sun J, Tong Z, Li B, Li G, Li W, Li J, Bellomo R. The prognostic value of the strong ion gap in acute pancreatitis. J Crit Care. 2016;36:140-5.

21. Hong W, Lin S, Zippi M, Geng W, Stock S, Zimmer V, Xu C, Zhou M. Highdensity lipoprotein cholesterol, blood urea nitrogen, and serum Creatinine can predict severe acute pancreatitis. Biomed Res Int. 2017;2017:1648385.

22. Zhou CL, Zhang CH, Zhao XY, Chen SH, Liang HJ, Hu CL, Chen NW. Early prediction of persistent organ failure by serum apolipoprotein A-l and highdensity lipoprotein cholesterol in patients with acute pancreatitis. Clin Chim Acta. 2018:476:139-45.

23. Peng YS, Chen YC, Tian YC, Yang CW, Lien JM, Fang JT, Wu CS, Hung CF, Hwang TL, Tsai YH, et al. Serum levels of apolipoprotein A-I and high-density lipoprotein can predict organ failure in acute pancreatitis. Crit Care. 2015;1.

\section{Publisher's Note}

Springer Nature remains neutral with regard to jurisdictional claims in published maps and institutional affiliations.

Ready to submit your research? Choose BMC and benefit from:

- fast, convenient online submission

- thorough peer review by experienced researchers in your field

- rapid publication on acceptance

- support for research data, including large and complex data types

- gold Open Access which fosters wider collaboration and increased citations

- maximum visibility for your research: over $100 \mathrm{M}$ website views per year

At BMC, research is always in progress.

Learn more biomedcentral.com/submissions 